\title{
Health in the Era of the Internet of Things: A Domain-Specific Modelling Language
}

\author{
Gero Strobel \\ University Duisburg-Essen \\ gero.strobel@uni-due.de
}

\begin{abstract}
Based on the cultural, social and technological development of the last hundred years, the pursuit of health driven by primal instincts has been transformed in most areas worldwide into a quasi de facto standard. But this security is deceptive. Not only the global developments of the last few months, but also creeping factors such as demographic change or the steadily growing world population are putting increasing pressure on the healthcare system. Today, more and more health care systems based on continuous demand for medical resources are increasingly reaching their limits. Technologies such as the Internet of Things are not a panacea, but offer great potential for increasing the flexibility and efficiency of healthcare systems. However, the development of such IoT-based healthcare systems requires a holistic understanding of the information technology as well as the domain itself. Against this background, the paper presents a domain-specific modelling language for IoT-based healthcare systems.
\end{abstract}

\section{Introduction}

Physical integrity has been the most basic human need since the beginning of time [20]. In the course of the cultural, technological and social development of the last hundred years, this need has increasingly become a standard in large parts of the world. However, this security is deceptive, as the global developments of recent months have shown [17]. In addition to the excessive burden of pandemics, developments such as the constantly growing population, an ageing society and the massive increase in widespread diseases are also putting a strain on the health system [3]. Due to the constantly increasing medical demand, resources such as infrastructure, skilled personnel but also simple medicines are becoming an increasingly valuable commodity, which will push traditional treatment methods to their limits in the future. The anamnesis and treatment of the cardiovascular system or chronic diseases, for example, requires continuous monitoring of medically relevant indication parameters, which leads to a commitment of technical but also humanitarian resources.

Especially during peak loads and in extreme situations, today's health care system reaches its limits, which patients worldwide have had to feel in recent months [17, 23]. Even patients in countries with a high income level no longer reduced these effects to disadvantages such as long waiting times or the effort of traveling, but had life-threatening consequences. In middle to low income countries, the often disastrous situation has worsened even further, so that in the worst case, treatment could not be given because the distance, overloaded or non-existent infrastructure made it impossible to visit a clinic or specialist [21, 23].

The Internet of Things, cloud computing and machine learning show great potential to reduce the steadily increasing pressure on the health care system and to establish decentralized supply systems. In particular, the use of smart products can help to reduce the effort and costs of standardized examination procedures. Central aspects here are the areas of health monitoring and ambient assisted living in which the use of IoT-based systems opens up new opportunities and possibilities for patients, their relatives and medical staff [12]. The central aspect here is the remote monitoring of medically relevant parameters, such as vital signs (e.g. blood pressure, pulse, etc.) or blood sugar levels. This does not only relieve the patients, but also offers the possibility to discharge them faster after an operation or to treat chronic diseases from home [9, 21, 29].

Furthermore, patients with a chronic disease, or whose vital signs need constantly to be measured and controlled, can be monitored comfortably from home, giving them a better quality of life [29]. Specifically in the field of ambient assisted living, technology offers new possibilities such as automatic medication reminders, fall alarms or even behavioral recognition, 
which offers elderly and disabled people, who in many cases are otherwise dependent on care personnel, more security, freedom and an increase in the quality of life [19].

Some differentiated approaches to IoT-based care are already present in the literature; however, many of these approaches are strongly domain-oriented (health monitoring, ambient assisted living, etc.) and technologically focused. To guarantee suitable IoTbased healthcare for patients, holistic information systems that adopt an overall systems perspective and integrate both system-relevant technological and human aspects are required. In order to be able to adopt such a perspective, however, it is essential to build on a uniform understanding of terms and a common language. Against this background, this paper examines the following research question:

How must a modeling language be designed to satisfy the demands of the smart health domain?

In the context of this publication, the research question is elaborated on the development of a domain-specific modelling language (DSML). Aim of the language is to foster the common understanding and provide a tool to develop more holistic IoT-based systems for the domain of smart health. The remainder of this paper is structured as follows. The paper opens with a description of the evolution of the healthcare domain, from telemedicine to smart health, based on technological developments. Then, we present a more detailed examination of the health information systems field. The applied research approach follows. Next, the developed domain-specific modelling language is discussed in detail. The paper concludes with comments on the limitations of the work and suggestions for future research.

\section{Evolution from e-Health to Smart Health}

The use of telecommunications and information technology to support medical processes and tasks is not in itself a new phenomenon. For the first time at the beginning of the 20th century, the term telemedicine was used to describe the use of information technology that enables both the auditory and visual exchange of information between medical professionals, medical service providers and patients over long distances [4, 5]. Over time, the term telehealth expanded the concept of telemedicine to include medical aspects that are not purely personal, such as public health, health education or even environmental health [16].
With the widespread use of the Internet, the term e-health first appeared at the beginning of the $21 \mathrm{st}$ century as a subcategory of classical health care, which can be defined as "an emerging field in the intersection of medical informatics, public health and business, referring to health services and information delivered or enhanced through the Internet and related technologies [...]" [8].

Due to ever more powerful hardware and the associated emergence of mobile devices, a growing trend towards mobile healthcare has developed over the years. The term mHealth was first coined and defined by Istepanian et al. as "mobile computing, medical sensor and communication technologies for healthcare "mobile computing, medical sensor and communication technologies for healthcare" [14, 15]. This in context relatively simple definitional description is still valid today and represents the essential characteristics of the paradigm independent of technological development [16]. Through the integration of sensors, actuators and smart products in all relevant areas of everyday life will be initiated new era of health care is comparable to the emergence of ehealth era based on the spread of the Internet. However, in contrast to patient-centered mHealth, Smart Health not only considers the patient as a system-relevant entity, but also his system environment and his interaction with it [25].

Detached from the scope of the system, these interaction possibilities only become possible through the range of capabilities of smart products and services. The range of capabilities extends from monitoring the environment and the system's own operating status using sensors, through the physical or electrical control of objects by actuators, to fully autonomous interaction with the environment [22]. Monitoring and controlling are essential for the domain of health. Here, developments in the field of sensor technology and IoT go hand in hand and allow a permanent further development of medical scanning based on single sensors, sensor networks, as well as implanted bimolecular sensors [16].

\section{Health Information Systems}

Information systems and their computer-aided support are indispensable today in all relevant areas of professional and everyday life. Even in system-critical domains, such as the health care system, an operative process without such systems is unthinkable today. This development began in the mid-1960s with the use of the first computer-aided system for creating and managing medical records in hospitals [1].

Irrespective of technological developments, today's health information systems (HIS) are still 
based on this basic idea of bringing together, aggregating and presenting all information relevant to the patient's healing process in a way that is appropriate for the addressee. These information systems are not just a piece of hardware or software, but represent socio-technical systems that represent technical, but above all human components in mostly very differentiated roles and with different objectives [32]. In general, health information systems can be defined as information systems specialized in the health sector, which, ,integrate data collection, processing, reporting, and use of the information necessary for improving health service effectiveness and efficiency through better management at all levels of health services " [18].

Health information systems are not an atomic, self-sufficient system, but a network of different subsystems, a so-called system of systems. Which in turn consists of a multitude of different individual systems (e.g. hospital information system). These subsystems in turn represent system networks that connect context-relevant systems such as laboratory information systems, radiology information systems, etc. [32].

\section{Research Method}

This paper is part of a multi-paper, design scienceoriented research project. The goal of the project is to develop a domain-specific information systems architecture and corresponding modelling language that includes modelling tools for the smart health domain.

The research presented in this paper are interim results and focused on the development and evaluation of the domain-specific language. The goal of the language development is to create a uniform basis for increasing common understanding within the domain and to translate essential domain-specific technical terms into technological concepts, so that users can focus primarily on the use of the language in the future and not be forced to reconstruct technical terms on their own [11].

DSMLs have established themselves as a methodological approach within the literature for this purpose, since they include all constructs relevant to the modeler and the domain in an appropriate, abstracted form, thus enabling modelers to create wellformed representations of the real world. These properties are not only reflected in the increased quality of the model, but also lead to a better understanding of the model itself $[6,11]$.

The starting point of this project's three-step language development effort was Frank's [11] DSML development approach, which is established in the conceptual modelling literature. In the first step, the essential components of the language, their dependencies and the corresponding modeling rules were defined as abstract syntax in the form of a metamodel (Figure 1) [6]. The extraction, aggregation of the corresponding concepts and also the development of the meta model were carried out and published in a preliminary study [27] based on an exploratory literature analysis of more than 6000 publications and the aggregation of 55 architecture approaches based on Webster and Watson [31], in combination with Vom Brocke et al. [30].

Based on this, the abstract concepts were concretized, defined and transformed into a concrete syntax in the form of a graphical notation (Figure 2). The goal of this step was to make the abstract linguistic concepts usable. In addition to the development of the language, its evaluation and demonstration of its usability are essential parts of language development [11]. In a third and final step, the developed DSML will be applied to a real-world smart health scenario, the myocardial infarction combined-device recovery enhancement (MiCORE) study and the associated Corrie Health platform for myocardial infarction patients at John Hopkins University $[13,26]$.The result of the development process is the Smart Health Modelling Language (SHML), a domain-specific language with which it is possible to model information systems, digital platforms or entire ecosystems with a focus on smart products and services within the smart health domain.

\section{Smart Health Modelling Language - A DSML for Smart Health}

\subsection{Abstract Syntax}

SHML takes a holistic, systemic perspective to guarantee suitable IoT-based healthcare for patients. This is only possible through the integration of system-relevant technological and human aspects. The meta model consists of four central components: a smart product, a smart service, a network and data.

Smart products are an integral part of the model. They consist of three basic components: a sensory component; connectivity, which enables the products to exchange data with other smart products or other system-relevant entities; and a physical device that encloses all the components [24].

Another central meta-model component represents the data available in the smart HIS. This data can be divided into smart data and medical data. Smart data include all data generated or measured by smart products and their sensors, and thus cover a diverse 
spectrum. Medical data can be differentiated into the areas of medical knowledge and health care records. Medical knowledge represents all knowledge of the domain collected by humans or machines, such as clinical studies. Meanwhile, healthcare records are personal data and, as the name suggests, include personal medical records, which may also contain a wide range of different data, such as anamnesis, information on vaccination status, laboratory values, $\mathrm{X}$-rays or even doctors' notes. In addition, information on insurance status, medical bills, medical appointments or even emergency data and living wills can be part of a healthcare record.

Smart services are the central contact point and interaction possibility for system users. In contrast to many other areas in which smart services are primarily consumed by end customers, there is a strong shift in the smart health area toward trained specialists and domain experts; this has resulted in a clearly broad spectrum of users and correspondingly broad requirements. Users within the domain can be divided into three groups: patients, caregivers and health professionals (HPs). The patient and his or her acquaintances and family members, who are subsumed under the term "caregivers" and who can access the patient's data with the patient's consent, take on the role of the end customer. By contrast, the user group of HPs (such as physicians, surgeons or nurses), who have a high degree of domain knowledge, must often make vital decisions in seconds based on the available data, but they also generate data.

In order for the individual entities of the system to communicate with each other, most IoT domains (i.e., smart home, smart farming, etc.) use two categories of network topologies, short-range and long-range. Within the health domain, the classification is extended by one category, with the so-called "Wireless Body Area Network" (WBAN). WBANs connect sensors and actuators that are placed very close to the body over long periods of time, or even permanently. In some cases, they are firmly implanted in the body. Short-range describes the use of technologies such as Wi-Fi, Bluetooth, ZigBee or RFID, which can cover a range from a few centimeters up to about 100 meters. Long-range protocols, which are used in the field and are based on existing infrastructures such as $5 \mathrm{G}$ or with the help of lowpower wide area networks specially set up for the application, can cover distances of several kilometers. However, for most of the application cases under consideration, a hybrid use of the different network topologies is appropriate.

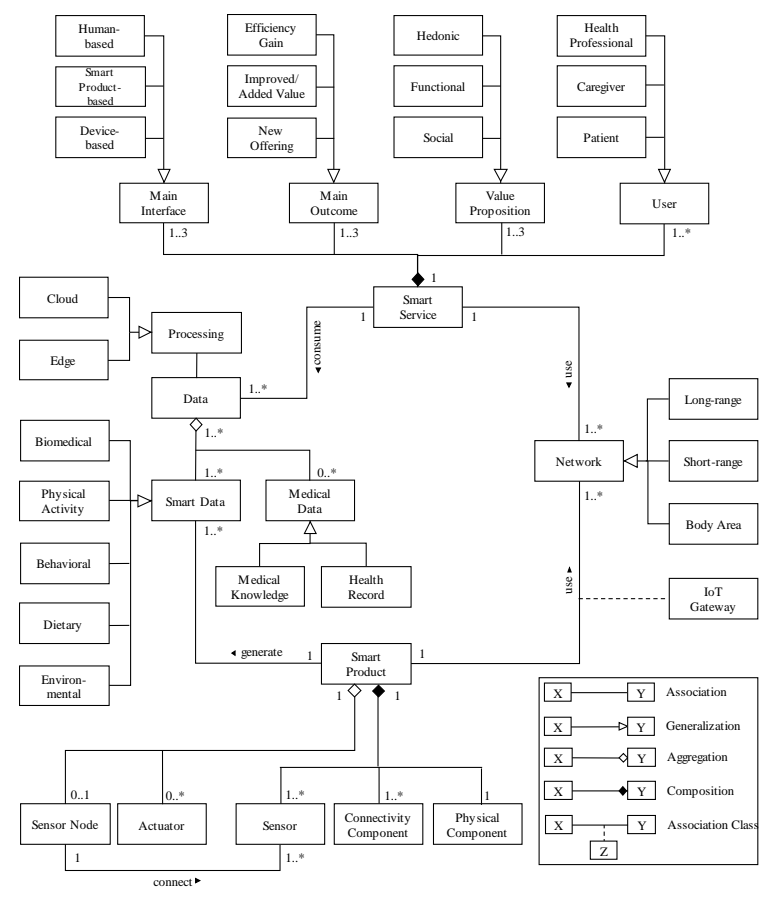

Figure 1. Abstract syntax [27]

\subsection{Concrete Syntax}

The concrete syntax of the SHML consists of both graphical and textual notations (Figure 2) that were developed based on established visualization guideline [2].

Basic elements of the language represent smart products and services. The element class of smart products is represented by circles, with the exception of the sensor node (rhombus). Depending on the range of capabilities, the elements can be enriched with up to three properties (processing, network, data) by appropriate annotations. In addition to complex smart products, individual sensors or actuators can be mapped in this way.

Smart services follow basically the same structure as smart products, but are represented in the language by a triangle. The model focuses on the service properties (main outcome, main interface, value proposition). Similar to smart products, the properties do not have to be mutually exclusive, so a smart service can simultaneously offer both a functional and hedonic added value for the user.

In addition to smart products and services, the syntax offers a wide range of different data types, making it possible not only to model abstract data flows, but also to specify exactly which data have been generated or exchanged, thus increasing the quality of the model itself. 


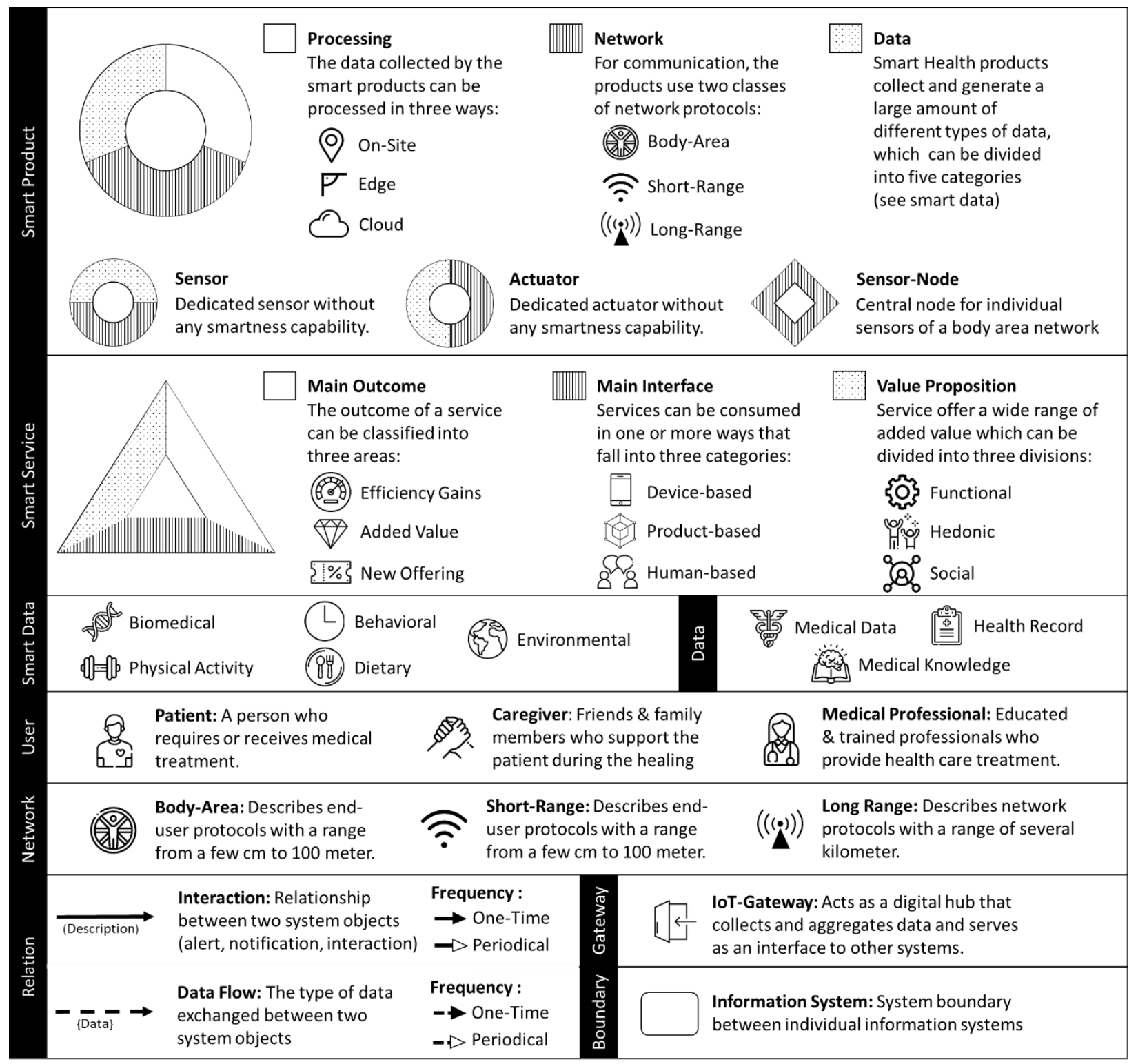

Figure 2. Concrete syntax

During the syntax's development, we took care not to develop the user as a generic element, but rather to define him/her with domain-specific roles.

Relationships among the elements can be represented with the relations on the data level, and on the interaction level (e.g. alert, notification, use), both once and periodically

\section{Demonstration and Evaluation}

The modelled scenario describes the Corrie digital health platform of John Hopkins University for patients after myocardial infarction (Figure 3) (Corrie Health) $[13,26]$.
The main focus of the platform is the support and monitoring of myocardial infarction patients during home rehabilitation, to prevent the occurrence of further myocardial events, which occur in about $20 \%$ of all patients within 30 days of discharge.

The following scenario describes a typical application case from a systemic perspective, with a focus on "Robert," a heart attack survivor who was familiarized with the corrie system before his discharge, received the technical equipment and is now in home rehabilitation. To monitor his vital signs, Robert uses his Apple Watch, which automatically transmits data such as stride rate and heart rate to the Corrie Health app (1). Furthermore, Robert measures 


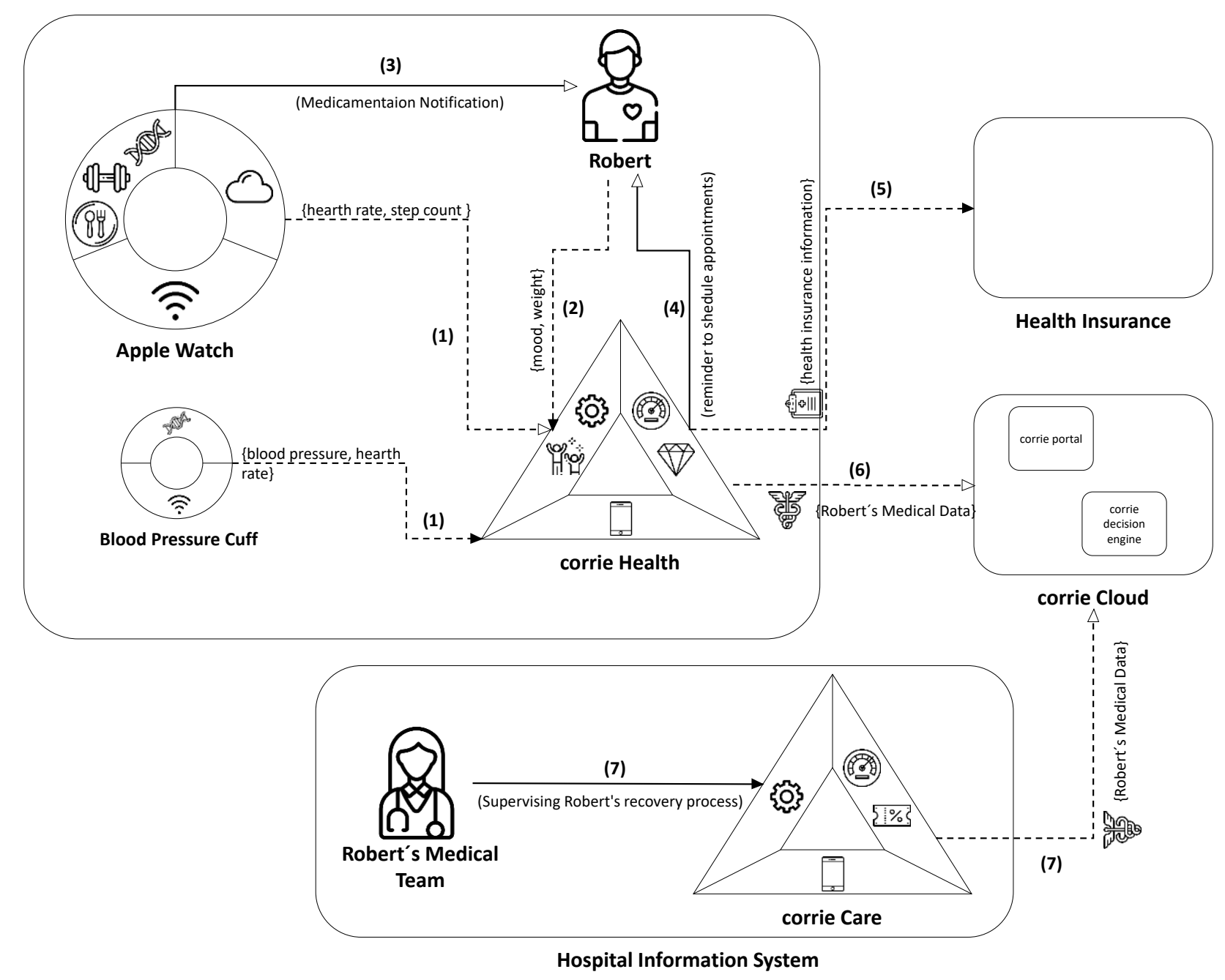

Figure 3. Evaluation of the SHML

his blood pressure in the morning and in the evening with a compatible blood pressure monitor (1). He manually enriches his vital data with information not measurable by the devices, such as his weight or current state of mind, using the Corrie Health app (2).

In addition to physical rehabilitation, independent medication at home and the continuation of medical check-ups are essential parts of his recovery program. For this purpose, the watch reminds him with visual, or even haptic, feedback to take his medication (3) and supports him in planning his check-up appointments based on the previously developed rehabilitation plan (4).

Further, the service enables the integration of a digital insurance card that can be used during visits to the doctor or in an emergency (5). The data Robert collects is transferred to the corrie cloud at regular intervals (6) and processed there. This enables Robert's doctors to monitor his rehabilitation process
(7) and intervene or adjust his rehabilitation program, if necessary.

\section{Conclusion and Outlook}

The use and integration of smart products will increase in the coming years as digitalization progresses. McKinsey predicts that, by 2023 , more than 43 billion intelligent devices will be in use in various domains of everyday life, such as smart homes, smart energy, smart cities or smart health [7].

For this reason, it is essential for modelers and system developers, especially in system-critical areas such as healthcare or the energy sector, not only to have a basic, independent but also a common understanding of intelligent products, their structure, interaction possibilities and range of services. This makes it possible for modelers and developers to participate in the emergence of new smart information 
systems or in the transformation process of existing systems.

Against this background, this paper offers a systematic and methodologically sound artifact based on a design-science-research oriented multi-paper study and the analysis of more than 6000 publications for the analysis, development and modelling of information systems in the form of a domain-specific modelling language for the domain of smart health.

The SHML can serve both as a tool for the analysis of existing information systems and as a conceptual tool for the development of new systems. The central focus is not only on the system-relevant components, but also on their relationship to each other. Although the research presented provides both theoretical and practical implications, it is not free of limitations. Especially in the context of a constantly changing and technology-driven domain like smart health, the presented DSML and its objects can only provide a snapshot of the current state of development. For this reason, it is essential to evaluate the language and its elements further in the context of future applications and, if necessary, adapt it to future developments of the domain.

The presented modelling language offers researchers a methodologically sound starting point for the development of dedicated solutions for the domain of smart health and a contact point for future research.

The central focus should be primarily on the development of an independent modeling tool for the presented DSML, with which not only researchers but also companies can productively use the language without great effort. Existing meta-modelling tools such as MEMO [10] or MetaEdit+ [28] could serve as a starting point for this development.

A further technological aspect that could be interesting in the context of the development of a corresponding modeling tool would be the porting of the tool from the second to the third dimension by using augmented reality. By enriching reality with data relevant for modelling or system analysis, new possibilities and added value arise for both the modeler and the model user. Based on the presented meta model it would also be conceivable to develop further specific models and languages for dedicated aspects like data flow within the systems.

In the next step, based on the results of this paper, a first prototypical implementation of the language for the Microsoft Hololens 2 is going to be developed.

Finally, the developed language offers new opportunities related to the development and modelling of information systems in the field of smart health and creates a conceptual basis for future research within the domain.

\section{References}

[1] Almunawar, M.N. and M. Anshari, Health Information Systems (HIS): Concept and Technology, 2012.

[2] Apfelbacher, R., A. Knöpfel, P. Aschenbrenner, and S. Preetz, FMC - Visualization Guidelines https://bit.ly/34DFzQR, accessed 11-10-2020.

[3] Baker, S.B., W. Xiang, and I. Atkinson, "Internet of Things for Smart Healthcare: Technologies, Challenges, and Opportunities", IEEE Access, 5, 2017, pp. 2652126544.

[4] Bashshur, R., J.H. Sanders, G. William, and G.W. Shannon, eds., Telemedicine: Theory and practice, Thomas, Springfield, Ill., 1997.

[5] Bashshur, R., G. Shannon, E. Krupinski, and J. Grigsby, "The Taxonomy of Telemedicine", Telemedicine Journal and e-health, 17(6), 2011, pp. 484-494.

[6] Becker, J., D. Beverungen, R. Knackstedt, H.P. Rauer, and D. Sigge, "On the Ontological Expressiveness of Conceptual Modeling Grammars for Service Productivity Management", Information Systems and e-Business Management, 12(3), 2014, pp. 337-365.

[7] Dahlqvist, F., M. Patel, A. Rajko, and J. Shulman, Growing opportunities in the Internet of Things https://mck.co/32rvTYi, accessed 11-10-2020.

[8] Eysenbach, G., "What is e-health?", Journal of medical Internet research, 3(2), 2001, E20.

[9] Fan, Y.J., Y.H. Yin, L.D. Xu, Y. Zeng, and F. Wu, "IoT-Based Smart Rehabilitation System", IEEE Transactions on Industrial Informatics, 10(2), 2014, pp. $1568-1577$.

[10] Frank, U., "MEMO: A Tool Supported Methodology for Analyzing and (Re-) Designing Business Information Systems", in Technology of Object-Oriented Languages ans Systems, R. Ege, M. Singh, and B. Meyer, Editors. 1994.

[11] Frank, U., "Domain-Specific Modeling Languages: Requirements Analysis and Design Guidelines", in Domain Engineering: Product Lines, Languages, and Conceptual Models, I. Reinhartz-Berger, A. Sturm, T. Clark, S. Cohen, and J. Bettin, Editors. 2013. Springer Berlin Heidelberg: Berlin, Heidelberg.

[12] Gope, P. and T. Hwang, "BSN-Care: A Secure IoTBased Modern Healthcare System Using Body Sensor Network", IEEE Sensors Journal, 16(5), 2016, pp. 1368 1376.

[13] Hung, G., W.E. Yang, F.A. Marvel, and S.S. Martin, "Mobile health application platform personalises and empowers the heart attack recovery patient experience in the hospital and at home for an underserved heart attack survivor", BMJ Case Reports CP, 13(2), 2020.

[14] Istepanian, R., E. Jovanov, and Y.-T. Zhang, "Introduction to the Special Section on M-Health: Beyond 
Seamless Mobility and Global Wireless Healthcare Connectivity", IEEE Transactions on Information Technology in Biomedicine, 8, 2005, pp. 405-414.

[15] Istepanian, R.S.H. and J.C. Lacal, "Emerging Mobile Communication Technologies for Health: Some Imperative Notes on M-health", in Proceedings of the 25th Annual International Conference of the IEEE Engineering in Medicine and Biology Society, Cancun, Mexico. 2003.

[16] Istepanian, R.S.H. and B. Woodward, M-health: Fundamentals and applications, Wiley IEEE Press, Hoboken, New Jersey, 2017.

[17] Kumar, A., K. Rajasekharan Nayar, and S.F. Koya, "COVID-19: Challenges and its consequences for rural health care in India", Public Health in Practice, 1, 2020, p. 100009.

[18] Lippeveld, T., R. Sauerborn, and C. Bodart, Design and Implementation of Health Information Systems, World Health Organization, Geneva, 2000.

[19] Lloret, J., L. Parra, M. Taha, and J. Tomás, "An architecture and protocol for smart continuous eHealth monitoring using 5G", Computer Networks, 129, 2017 , pp. 340-351.

[20] Maslow, A.H., "A theory of human motivation", Psychological Review, 50(4), 1943, pp. 370-396.

[21] Pathinarupothi, R.K., M.V. Ramesh, and E. Rangan, "Multi-layer Architectures for Remote Health Monitoring", in Proceedings of the IEEE 18th International Conference on e-Health Networking, Applications and Services, Munich, Germany. 2016.

[22] Porter, M. and J. Heppelmann, "How Smart, Connected Products Are Transforming Competition", Harvard Business Review, 92(11), 2014, pp. 64-88.

[23] Roy, D., S. Tripathy, S.K. Kar, N. Sharma, S.K. Verma, and V. Kaushal, "Study of knowledge, attitude, anxiety \& perceived mental healthcare need in Indian population during COVID-19 pandemic", Asian journal of psychiatry, 51, 2020, p. 102083.

[24] Schiller, B., T. Brogt, J.M.P. Schuler, and G. Strobel, "Can Self-Tracking Solutions Help with Understanding Quality of Smart, Connected Products?", in Proceedings of the 26th European Conference on Information Systems, Portsmouth, England. 2018.

[25] Solanas, A., C. Patsakis, M. Conti, I. Vlachos, V. Ramos, F. Falcone, O. Postolache, P. Perez-martinez, R. Pietro, D. Perrea, and A. Martinez-Balleste, "Smart health: A context-aware health paradigm within smart cities", IEEE Communications Magazine, 52(8), 2014, pp. 74-81.

[26] Spaulding, E.M., F.A. Marvel, M.A. Lee, W.E. Yang, R. Demo, J. Wang, H. Xun, L. Shah, D. Weng, O.E. Fashanu, J. Carter, J. Sheidy, R. McLin, J. Flowers, M. Majmudar, E. Elgin, V. Vilarino, D. Lumelsky, V. Bhardwaj, W. Padula, J.K. Allen, and S.S. Martin, "Corrie Health Digital Platform for Self-Management in Secondary Prevention After Acute Myocardial Infarction",
Circulation. Cardiovascular quality and outcomes, 12(5), 2019.

[27] Strobel, G. and J. Perl, "Health in the Era of the Internet of Things - A Smart Health Information System Architecture", in Proceedings of the 24nd Pacific Asia Conference on Information Systems, United Arab Emirates. 2020 .

[28] Tolvanen, J.-P. and M. Rossi, "MetaEdit+", in Proceedings of the 18th annual ACM SIGPLAN conference on Object-oriented programming, systems, languages, and applications, R. Crocker and G.L. Steele, Editors, Anaheim, CA, USA. 2003.

[29] Velasco, C.A., Y. Mohamad, and P. Ackermann, "Architecture of a Web of Things EHealth Framework for the Support of Users with Chronic Diseases", in Proceedings of the 7th International Conference on Software Development and Technologies for Enhancing Accessibility and Fighting Info-Exclusion. 2016: New York, NY, USA.

[30] Vom Brocke, J., A. Simons, B. Niehaves, K. Riemer, R. Plattfaut, and A. Cleven, "Reconstructing the giant: On the importance of rigour in documenting the literature search process", in Proceedings of the 17th European Conference on Information Systems, Verona, Italy. 2009.

[31] Webster, J. and R.T. Watson, "Analyzing the past to prepare for the future: Writing a literature review", MIS Quarterly, 2002, pp. xiii-xxiii.

[32] Winter, A., R. Haux, E. Ammenwerth, B. Brigl, N. Hellrung, and F. Jahn, Health Information Systems, Springer London, London, 2011. 